\title{
mRCC-Patienten profitieren von Cabozantinib
}

\author{
Eine neue Therapieoption beim metastasierten Nierenzellkarzinom (mRCC) \\ mit VEGFR-Inhibitor-Resistenz bietet der Multikinasehemmer Cabozantinib. In \\ einer Phase-III-Studie wurde er mit Everolimus verglichen.
}

_ ast alle Patienten mit mRCC werden

- 8-11 Monate nach der Erst- bzw. 3-5 Monate nach der Zweitlinientherapie resistent gegen VEGF-Hemmer. Hier verlängert der mTOR-Inhibitor Everolimus im Vergleich zu Placebo das progressionsfreie Überleben (PFS; median 4,9 vs. 1,9 Monate). Ob der neue Multikinase-

hemmer Cabozantinib wirksamer ist, war Fragestellung einer Phase-III-Studie.

Randomisiert erhielten 658 Patienten $60 \mathrm{mg} / \mathrm{d}$ Cabozantinib oder $10 \mathrm{mg} / \mathrm{d}$ Everolimus. Beim primären Endpunkt PFS, zu dem die Daten der ersten 375 Patienten ausgewertet wurden, erwies sich $\mathrm{Ca}$ bozantinib als überlegen (median 7,4 vs.

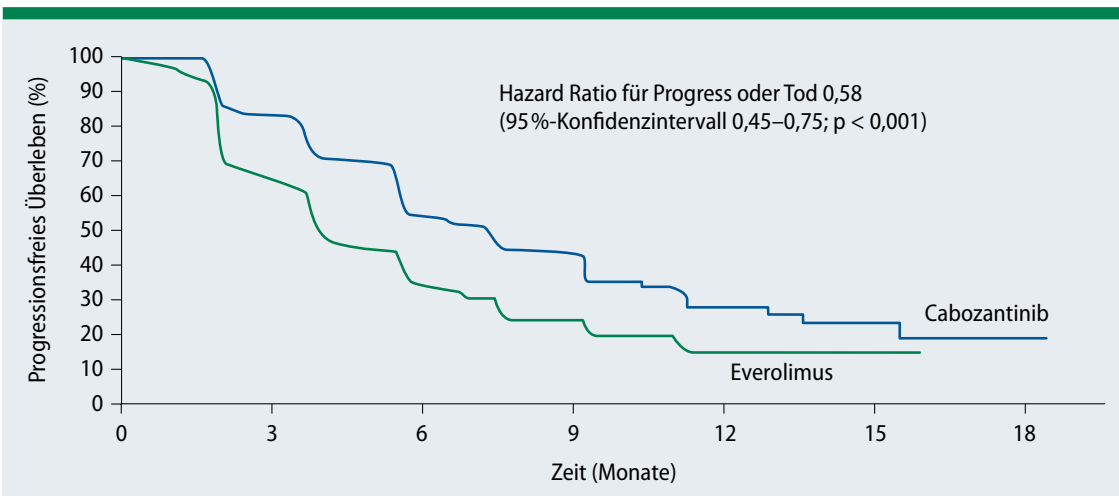

Abb. 1: Unter Cabozantinib lebten Patienten länger progressionfrei als unter Everolismus.

\section{RCC: Kardiovaskuläres Risiko unter Anti-VEGF-TKI}

\section{Das kardiovaskuläre Risiko unter den Tyrosinkinasehemmern (TKI) Sorafe- nib und Sunitinib wurde in einer po- pulationsbasierten Beobachtungs- studie bei älteren Patienten mit Nie- renzellkarzinom (RCC) untersucht.}

nsgesamt 670 Patienten aus dem SEER (Surveillance, Epidemiology, and End Results)-Register, die mindestens 66 Jahre alt waren und bei denen zwischen 2000 und 2009 ein RCC diagnostiziert worden war, hatten Sunitinib und/oder Sorafenib erhalten, 111 beide Substanzen. Ein Drittel von ihnen war 75 Jahre oder älter, mehr als die Hälfte hatte eine oder mehrere Komorbiditäten. Unerwünschte kardiovaskuläre (KV) Ereignisse wie Herzinsuffizienz und Kardiomyopathie, akuter Myokardinfarkt,
Schlaganfall und KV-Todesfälle traten bis Dezember 2010 bei 171 Patienten auf, die Sunitinib und/oder Sorafenib erhalten hatten (Gesamtinzidenzrate 1,02, Inzidenz für Herzinsuffizienz und Kardiomyopathie 0,87, für akuten Myokardinfarkt 0,14 und für Schlaganfall 0,14 pro 1.000 Personentage). Im Vergleich zu 788 Patienten mit fortgeschrittenem RCC, die keines der beiden Medikamente erhielten, war die Anwendung von Sunitinib oder Sorafenib mit einem erhöhten Risiko für KV-Ereignisse assoziiert (Hazard Ratio [HR] 1,38). Am stärksten nahm das Schlaganfallrisiko zu (HR insgesamt 2,84, für Sorafenib allein 5,30 und für Sunitinib allein 2,28). Das Risiko für Herzinsuffizienz und Kardiomyopathie stieg lediglich unter Sunitinib an (HR 1,53). Das Herzinfarktrisiko
3,8 Monate) (Abb. 1). Unter Cabozantinib betrug die Ansprechrate $21 \%$, unter Everolimus 5\% ( $\mathrm{p}<0,001)$. Der Vorteil beim PFS für Cabozantinib zeigte sich in allen Subgruppen, unabhängig von z. B. Risikokategorie und vorangegangener VEGFR-Inhibitor-Behandlung. Die geplante Interimsanalyse ergab einen Trend zu einem verlängertem Gesamtüberleben durch Cabozantinib.

Dosisreduktionen waren jedoch bei rund $60 \%$ der Patienten in der Cabozantinib-Gruppe - v.a. wegen schwerer Hypertonie, Diarrhö und Fatigue- und bei $25 \%$ in der Everolimus-Gruppe - v.a. wegen schwerer Anämien und Fatigue nötig. Studienabbrüche aufgrund von Toxizitäten waren in den Gruppen mit $9 \%$ bzw. $10 \%$ vergleichbar häufig.

Fazit: Patienten mit mRCC, die gegen klassische Antiangiogenetika wie Bevacizumab, Sunitinib oder Sorafenib resistent geworden sind, profitieren möglicherweise bei PFS und Gesamtüberleben eher von Cabozantinib als vom bisherigen Standard Everolimus. Barbara Kreutzkamp

Choueiri TK et al. Cabozantinib versus everolimus in advanced renal-cell carcinoma. N Engl J Med. 2015;373(19):1814-23.

war bei Behandlung mit einer der beiden Substanzen leicht, aber nicht signifikant erhöht. Deutlich mit einem erhöhten Schlaganfallrisiko assoziiert waren auch zunehmendes Alter und zunehmende Komorbidität. Subgruppenanalysen zeigten, dass der Effekt der TKI auf das Schlaganfallrisiko bei Patienten bis zu 74 Jahren (HR 6,94) deutlich stärker war als bei den älteren (HR 1,33).

Fazit: Sunitinib und Sorafenib waren in dieser Untersuchung mit einem erhöhten Risiko für KV-Ereignisse assoziiert, v.a. für Schlaganfall. Auch, wenn das absolute Risiko gering ist, sollten Nutzen und Risiken abgewogen und ggfs. frühzeitig der Entwicklung KV-Ereignisse gegengesteuert werden. Judith Neumaier

Jang S et al. Cardiovascular Toxicity After Antiangiogenic Therapy in Persons Older Than 65 Years With Advanced Renal Cell Carcinoma. Cancer. 2016;122(1):124-30. 